\title{
Atributos químicos de um Latossolo Amarelo sob diferentes sistemas de manejo
}

\author{
Liliane Pereira Campos ${ }^{(1)}$, Luiz Fernando Carvalho Leite ${ }^{(2)}$, Giovana Alcântara Maciel ${ }^{(3)}$, \\ Bruna de Freitas Iwata ${ }^{(4)}$ e Júlio César Azevedo Nóbrega ${ }^{(1)}$
}

\begin{abstract}
(1)Universidade Federal do Piauí (UFPI), Campus Profa. Cinobelina Elvas, BR 135, Km 03, CEP $64900-000$ Bom Jesus, PI. E-mail: licalivre@hotmail.com, jnobrega@ufpi.br (2)Embrapa Meio-Norte, Avenida Duque de Caxias, 5.650, Bairro Buenos Aires, CEP 64006-220 Teresina, PI. E-mail: luizf@cpmn.embrapa.br ${ }^{(3)}$ Embrapa Cerrados, BR 020, Km 18, CEP $73310-970$ Planaltina, DF. E-mail: giovana.maciel@cpac.embrapa.br (4)UFPI, Campus Ministro Petrônio Portella, s/no, Bairro Ininga, CEP 64049-550 Teresina, PI. E-mail: iwatameioambiente@gmail.com
\end{abstract}

Resumo - O objetivo deste trabalho foi avaliar os atributos químicos de um Latossolo Amarelo sob diferentes sistemas de manejo do solo, no cerrado piauiense. Foram avaliados quatro sistemas de manejo: plantio convencional por três anos; plantio direto por três anos (PD3) e cinco anos (PD5), com uso de milheto como cultivo de cobertura; e plantio direto por nove anos (PD9), dos quais sete com uso de milheto e dois com forrageira. Utilizou-se área de cerrado nativo como referência. As amostras do solo foram coletadas em períodos chuvosos e secos, nas camadas $0,00-0,05,0,05-0,10,0,10-0,20$ e $0,20-0,40 \mathrm{~m}$, para determinação de $\mathrm{pH}, \mathrm{Al}^{3+}, \mathrm{H}+\mathrm{Al}, \mathrm{Ca}^{2+}, \mathrm{Mg}^{2+}, \mathrm{K}^{+}, \mathrm{P}$ disponível, carbono orgânico total (COT), soma de bases (SB), capacidade de troca de cátions efetiva ( $\mathrm{t}$ ) e potencial $(\mathrm{T})$, e saturação por bases $(\mathrm{V})$ e por alumínio $(\mathrm{m} \%)$. O sistema PD9 apresentou maiores valores de $\mathrm{pH}$ e menores de $\mathrm{Al}^{3+}, \mathrm{H}+\mathrm{Al}$ e $\mathrm{m} \%$. Observaram-se maiores valores de $\mathrm{Ca}^{2+}, \mathrm{K}^{+}$, $\mathrm{SB}, \mathrm{t}, \mathrm{T}, \mathrm{V}$ e P sob PD5 e PD9, até 0,20 m. Os maiores valores de COT foram verificados sob PD5 e PD9, exceto na camada de 0,00-0,05 m. O acúmulo de material orgânico associado ao uso de forrageira em PD favorece o aumento dos teores de COT nas camadas mais profundas do solo, no período seco.

Termos para indexação: carbono orgânico total, fertilidade do solo, matéria orgânica do solo, plantio convencional, plantio direto, qualidade do solo.

\section{Chemical attributes in a Xanthic Ferralsol under different tillage systems}

\begin{abstract}
The objective of this work was to evaluate the chemical attributes in a Xanthic Ferralsol under different tillage systems in the cerrado of Piauí State, Brazil. Four tillage systems were evaluated: three-year-old conventional tillage; no-tillage with three (NT3) and five years old (NT5), using millet as cover crop; and no-tillage with nine years old (NT9), of which seven years used millet as cover crop and two used forage. A native cerrado area was taken as reference. Soil samples were collected in wet and dry seasons at soil depths, 0.00-0.05, 0.05-0.10, 0.10-0.20 and 0.20-0.40 m, and analyzed for $\mathrm{pH}, \mathrm{Al}^{3+}, \mathrm{H}+\mathrm{Al}, \mathrm{Ca}^{2+}, \mathrm{Mg}^{2+}, \mathrm{K}^{+}$, sum of bases (SB), effective ( $(\mathrm{t})$ and potential $(\mathrm{T})$ cation exchangeable capacity, base (BS) and $\mathrm{Al}^{3+}$ saturation $(\mathrm{m} \%)$, available $\mathrm{P}$ and total organic carbon (TOC). The system NT9 showed the highest values of pH and lowest of $\mathrm{Al}^{3+}, \mathrm{H}+\mathrm{Al}$, and $\mathrm{m} \%$. The highest values for $\mathrm{Ca}^{2+}, \mathrm{K}, \mathrm{SB}, \mathrm{t}, \mathrm{T}, \mathrm{V} \%$ and $\mathrm{P}$, were observed under NT5 and NT9, until $0.20 \mathrm{~m}$ depth. The highest TOC contents were verified also under NT5 and NT9, except for 0.00-0.05 m soil depth. Organic matter accumulation associated with pasture under no-till increases TOC content at deeper soil layers in the dry period.
\end{abstract}

Index terms: total organic carbon, soil fertility, soil organic matter, conventional tillage, no-tillage, soil quality.

\section{Introdução}

A presença de nutrientes e o manejo adequado do solo são aspectos fundamentais que garantem a melhoria da qualidade dos solos, principalmente no caso de agrossistemas em regiões de clima tropical (Balota et al., 2004). Assim, práticas agrícolas que reduzam a perda de solo por cultivos intensivos e mantenham a presença de plantas de cobertura na entressafra, podem favorecer a sustentabilidade da produção em região sob cerrado (Boer et al., 2007).

A predominância do monocultivo associado a práticas agrícolas inadequadas, com excesso de mobilização do solo, tem comprometido o crescimento da produtividade e resultado na degradação do solo e do ambiente. A realização de estudos que contemplem a dinâmica de nutrientes de solos sob cerrado e o manejo da fertilidade com o crescimento e desenvolvimento

Pesq. agropec. bras., Brasília, v.46, n.12, p.1681-1689, dez. 2011 
de plantas podem otimizar o uso dos insumos, fertilizantes e corretivos, e tornar o sistema de cultivo ambientalmente sustentável (Goedert \& Oliveira, 2007).

Entre as melhorias constatadas na fertilidade do solo com uso de sistemas de manejo que não contemplam revolvimento do solo, destacam-se as alterações químicas em sistemas de plantio direto (PD). Este sistema é caracterizado pela formação de um ambiente orgânico que favorece a preservação da umidade e da fertilidade do solo, e que facilita a difusão do fósforo (P) na solução do solo e sua absorção pelas plantas (Gatiboni et al., 2007). Além disso, o uso de plantas de cobertura de solo na entressafra pode promover a liberação de ácidos orgânicos solúveis em água, capazes de complexar o alumínio trocável, mobilizar o cálcio e o magnésio (Zambrosi et al., 2008) e reter o potássio, evitando sua perda por lixiviação (Rosolem et al., 2003, Boer et al., 2007).

Aimplantação do PD em regiões de cerrado requer um conjunto de técnicas integradas que incluem: calagem em profundidade, adubação corretiva de macro e micro nutrientes e rotação de culturas (Lopes et al., 2004). No entanto, para o total sucesso do PD, é necessária a rotação de culturas entre gramíneas e leguminosas, uma vez que as espécies vegetais diferem entre si no que se refere a morfologia, quantidade e qualidade de resíduos remanescentes da colheita, eficiência de absorção de íons e exploração de diferentes profundidades de solo pelo sistema radicular (Santos et al., 2009).

Estudos comprovam que o PD associado ao uso de pastagens perenes, além de reduzir a decomposição da palhada em regiões de clima tropical, favorece a estrutura físico-química do solo (Fontana et al., 2006; Salton et al., 2008), a macrofauna edáfica do solo (Marchão et al., 2009) e, pela grande quantidade de material orgânico que acumula, pode funcionar como dreno de $\mathrm{CO}_{2}$ para a atmosfera (Nicoloso et al., 2008).

No cerrado do Estado do Piauí, considerada a última fronteira agrícola do Estado, com cerca de 12 milhões de hectares (Aguiar \& Monteiro, 2005), são incipientes os trabalhos com sistemas integrados de produção de grãos e pastagens e seu efeito na fertilidade do solo.

O objetivo deste trabalho foi avaliar os atributos químicos de um Latossolo Amarelo sob diferentes sistemas de manejo do solo, no cerrado do Piauí.

\section{Material e Métodos}

O trabalho foi realizado na Fazenda São Marcos, Município de Bom Jesus, a 0909'59"S, 4506'43"W, a $481 \mathrm{~m}$ de altitude, na região de cerrado da Serra do Quilombo, no sudoeste piauiense, Nordeste do Brasil. O clima da região, segundo a classificação de Köppen, é do tipo Aw, quente e semiúmido. A temperatura média anual é de $27^{\circ} \mathrm{C}$, com precipitação pluvial média anual de $1.000 \mathrm{~mm}$ e estação chuvosa de outubro a abril, com o trimestre mais chuvoso de janeiro a março, com ocorrência de veranicos. O solo é classificado como Latossolo Amarelo distrófico, de classe textural franco-argilo-arenosa (Santos et al., 2006) para as camadas de 0,00-0,05 m, 0,050,10 m, 0,10-0,20 m e 0,20-0,40 m, com média de $451,88,190,55,68,75$ e $288,82 \mathrm{~g} \mathrm{~kg}^{-1}$ de areia grossa, areia fina, silte e argila, respectivamente.

Foram avaliados quatro sistemas de manejo do solo, além de uma área de transição com fitofisionomia de campo cerrado e cerradão de cerrado nativo $(\mathrm{CN})$, usada como referência. Foram escolhidos talhões a cada $2 \mathrm{~km}$, conduzidos nos seguintes sistemas (Tabela 1): 1) plantio convencional por três anos (PC3) - após o desmate da área, o preparo do solo foi feito com uso de grade pesada, intermediária e niveladora, e cultivouse arroz por dois anos e fez-se o cultivo de soja com adubação de acordo com a necessidade da cultura no ano agrícola de 2009/2010, após correção com $2 \mathrm{Mg} \mathrm{ha}^{-1}$ de calcário e $300 \mathrm{~kg} \mathrm{ha}^{-1}$ de gesso agrícola; 2 e 3) plantio direto por três (PD3) e cinco anos (PD5) após uso de sistema convencional, tendo-se utilizado o milheto para a formação de palhada, fez-se o cultivo de soja no PD3 e o cultivo em rotação de soja/soja/milho/ soja/soja, no PD5; 4) plantio direto por nove anos (PD9) - após seis anos de plantio convencional, utilizou-se o PD, estabelecido por sete anos com uso de milheto como cultivo de cobertura do solo, com a rotação de soja/soja/soja/milho/milho/soja/milho, sendo que, após a colheita do milho, utilizou-se a forrageira Urochloa brizantha (Syn. Brachiaria brizantha), cultivar MG-5, para a formação da palhada e posterior cultivo de soja, no ano agrícola 2009/2010.

A primeira amostragem do solo foi feita em janeiro de 2010 (período chuvoso), durante a fase de desenvolvimento vegetativo da cultura da soja. Escolheu-se aleatoriamente uma área de 1,5 ha dentro de cada sistema, onde foram abertas oito minitrincheiras por sistema, com cinco repetições, numa diagonal 
com $0,60 \mathrm{~m}$ de profundidade, $0,60 \mathrm{~m}$ de largura e 0,60 m de comprimento, nas entrelinhas dos plantios. Foram retiradas amostras de quatro profundidades $(0,00-0,05 \mathrm{~m}, 0,05-0,10 \mathrm{~m}, 0,10-0,20 \mathrm{~m}$ e 0,20 $0,40 \mathrm{~m}$ ), das quais oito amostras simples, para formar uma composta, no total de 20 amostras por sistema. Todos os pontos foram georreferenciados e serviram para direcionar a segunda coleta, realizada em julho de 2010 (período seco), conforme os critérios da primeira amostragem.

As amostras de solo foram secadas ao ar, destorroadas, maceradas e passadas em peneira de $2 \mathrm{~mm}$ de abertura de malha (TFSA), para a determinação dos atributos químicos $\mathrm{pH}, \mathrm{K}^{+}, \mathrm{P}, \mathrm{Ca}^{2+}, \mathrm{Mg}^{2+}, \mathrm{Al}^{3+} \mathrm{e} \mathrm{H}+\mathrm{Al}$, conforme Silva (1999). A matéria orgânica do solo (MOS) foi determinada pelo método indireto do carbono orgânico total (COT), conforme descrito por Yeomans \& Bremner (1988). A partir dos resultados, foram calculadas: soma de bases (SB), capacidade de troca de cátions a $\mathrm{pH}$ 7,0 $(\mathrm{T})$, percentagem de saturação por bases (V), capacidade de troca de cátions a $\mathrm{pH}$ natural (t) e percentagem de saturação por alumínio $(\mathrm{m} \%)$, conforme descrições feitas por Ribeiro et al. (1999).

Os dados foram submetidos à análise de variância, e as médias foram comparadas pelo teste de Tukey, a 5\% de probabilidade, pelo programa estatístico Assistat (Silva \& Azevedo, 2009).

\section{Resultados e Discussão}

Os maiores valores de $\mathrm{pH}$ foram observados no solo sob PD9, nas camadas de $0,00-0,05,0,05-0,10$ e 0,10-0,20 m, embora, nesta última, somente no período chuvoso (Tabela 2). Resultados semelhantes foram observados por Matias et al. (2009), também no cerrado do Piauí, que relataram que o aumento de
pH pela adição de calcário, aplicado à superfície do solo ou com incorporação, não influenciou a correção da acidez do subsolo, na camada de 0,20-0,40 m, em sistema sob PD. Para a camada de $0,20-0,40 \mathrm{~m}$, maiores valores de $\mathrm{pH}$ foram observados no solo sob $\mathrm{CN}$, em ambos os períodos. Sousa et al. (2007) afirmam que, em condições de acúmulo de MOS em estágio final de mineralização, a oxidação libera elétrons para a solução do solo, o que propicia o aumento de $\mathrm{pH}$, mesmo em profundidade.

$\mathrm{O} \mathrm{CN}$ apresentou maiores teores de $\mathrm{Al}^{3+}$, em comparação aos sistemas de manejo, até a profundidade de $0,20 \mathrm{~m}$, no período chuvoso (Tabela 2). Carneiro et al. (2009) analisaram vários tipos de solos, em áreas sob vegetação de cerrado, e observaram maiores teores de $\mathrm{Al}^{3+}$ e menores de $\mathrm{Ca}^{2+}, \mathrm{Mg}^{2+}$ e $\mathrm{P}$, em comparação às áreas manejadas, em razão do baixo $\mathrm{pH}$ e da não correção e adubação do solo, originalmente distrófico. No entanto, menores valores foram observados no solo entre os sistemas PD, nas camadas de 0,00-0,05 m, no período chuvoso, e $0,20-0,40 \mathrm{~m}$, no período seco. A redução de $\mathrm{Al}^{3+}$ nos sistemas decorre dos efeitos da calagem; porém, os sistemas associados ao PD apresentaram menores teores de $\mathrm{Al}^{3+}$ do que o PC3. Esse resultado pode ser consequência do maior efeito da complexação do $\mathrm{Al}^{3+}$ proporcionada pelos compostos orgânicos, os quais, por sua vez, são resultantes do acúmulo de MOS nestes sistemas (Anghinoni, 2007).

Quanto aos resultados de $\mathrm{H}+\mathrm{Al}$, os maiores valores foram observados no $\mathrm{CN}$, na camada de 0,00 0,05 m, entre os períodos chuvoso e seco (Tabela 2). Nas demais camadas e períodos, valores superiores foram observados nos sistemas PD5, nas camadas de $0,05-0,10 \mathrm{~m}$ e $0,10-0,20 \mathrm{~m}$, e PD5 e PD9 na camada de $0,20-0,40 \mathrm{~m}$. A presença de material orgânico no $\mathrm{CN}$, na primeira camada, bem como nos sistemas de

Tabela 1. Histórico de uso e manejo de Latossolo Amarelo, na região do Cerrado, no sudoeste piauiense.

\begin{tabular}{|c|c|c|c|c|c|c|c|c|c|c|}
\hline \multirow[t]{2}{*}{ Sistemas de uso e manejo } & \multicolumn{5}{|c|}{ Plantio convencional } & \multicolumn{5}{|c|}{ Plantio direto } \\
\hline & $\begin{array}{l}\text { Período } \\
\text { (ano) }\end{array}$ & $\begin{array}{l}\text { Culturas } \\
\text { anuais }^{(1)}\end{array}$ & $\begin{array}{l}\text { Calcário } \\
\left(\mathrm{Mg} \mathrm{ha}^{-1}\right)\end{array}$ & $\begin{array}{c}\text { Gesso } \\
\left(\mathrm{kg} \mathrm{ha}^{-1}\right)\end{array}$ & $\begin{array}{l}\text { SS-KCl }{ }^{(4)} \\
\left(\mathrm{kg} \mathrm{ha}^{-1}\right)\end{array}$ & $\begin{array}{l}\text { Período } \\
\text { (ano) }\end{array}$ & $\begin{array}{l}\text { Culturas } \\
\text { anuais }^{(2)}\end{array}$ & $\begin{array}{l}\text { Calcário } \\
\left(\mathrm{Mg} \mathrm{ha}^{-1}\right)\end{array}$ & $\begin{array}{c}\text { Gesso } \\
\left(\mathrm{kg} \mathrm{ha}^{-1}\right)\end{array}$ & $\begin{array}{c}{\mathrm{MAP}-\mathrm{KCl}^{(5)}}_{\left(\mathrm{kg} \mathrm{ha}^{-1}\right)} \\
\end{array}$ \\
\hline Cerrado $(\mathrm{CN})$ & - & - & - & - & - & - & - & - & - & - \\
\hline Plantio Convencional (PC3) & $2007 / 08$ a $2009 / 10$ & A A S & 2 & 300 & $400-180$ & - & - & - & - & - \\
\hline Plantio direto (PD3) & $2003 / 04$ a $2005 / 06$ & A S S & $\mathrm{NC}^{(3)}$ & - & $\mathrm{NC}$ & $2006 / 07$ a $2009 / 10$ & $\mathrm{Mt} / \mathrm{S}, \mathrm{Mt} / \mathrm{S}, \mathrm{Mt} / \mathrm{S}$ & 2 & 300 & $180-180$ \\
\hline Plantio direto (PD5) & $1999 / 00$ a $2003 / 04$ & A S S S S & $\mathrm{NC}$ & - & $\mathrm{NC}$ & $2004 / 05$ a $2009 / 10$ & $\mathrm{Mt} / \mathrm{S}, \mathrm{Mt} / \mathrm{S}, \mathrm{Mi}, \mathrm{S}, \mathrm{S}$ & 1,3 & - & $180-180$ \\
\hline Plantio direto (PD9) & $1994 / 96$ a $2000 / 01$ & AA S S S S & $\mathrm{NC}$ & - & $\mathrm{NC}$ & $2001 / 02$ a $2009 / 10$ & $\begin{array}{c}\mathrm{Mt} / \mathrm{S}, \mathrm{Mt} / \mathrm{S}, \mathrm{Mt} / \mathrm{S}, \\
\mathrm{M}, \mathrm{M}, \mathrm{S}, \mathrm{M}, \mathrm{Bb}, \mathrm{Bb} / \mathrm{S}\end{array}$ & 1 & - & $180-180$ \\
\hline
\end{tabular}

(1) A, arroz de sequeiro; S, soja de sequeiro. (2) Mi, milho de sequeiro; Mt, milheto, plantio após colheita de soja; Bb, Urochloa brizantha, cv. 'MG 5', plantio após colheita do milho. ${ }^{(3)} \mathrm{NC}$, correção e adubação, conforme necessidade da cultura. ${ }^{\left({ }^{4}\right)} \mathrm{SS}-\mathrm{KCl}$, superfosfato simples-cloreto de potássio. ${ }^{(5)} \mathrm{MAP}-\mathrm{KCl}$, monoamônio fosfato-cloreto de potássio. 
manejo com mais tempo sob PD, pode ter contribuído para complexação de $\mathrm{H}^{+}$e $\mathrm{Al}^{3+}$ livres. Estudo realizado por Zambrosi et al. (2008), em Ponta Grossa, PR, num Latossolo sob PD, em área de pastagem nativa, mostraram que o $\mathrm{Al}^{3+}$ ocorreu, predominantemente, complexado pelo carbono orgânico dissolvido e pelo fluoreto, e, em baixíssimas proporções, na forma livre $\mathrm{Al}^{3+}$, mesmo em condições de alta acidez.

Os valores de $\mathrm{m} \%$ variaram de $3,7 \%$ na camada de 0,00-0,05 m, para o PD9 no período chuvoso, a $96 \%$ na camada $0,10-0,20 \mathrm{~m}$, para o $\mathrm{CN}$ no período seco (Tabela 2), e o uso de corretivos reduziu a acidez e a saturação por $\mathrm{Al}^{3+}$ até a camada $0,00-0,20 \mathrm{~m}$, considerada muito alta $(\mathrm{m} \geq 65 \%)$ quando se trata de $\mathrm{CN}$. A diminuição de $\mathrm{m} \%$ pode ser devida ao aumento

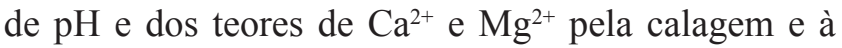
complexação de $\mathrm{Al}^{3+}$ por compostos orgânicos (Perin et al., 2003; Nolla \& Anghinoni, 2006).
Houve aumento significativo nos teores de bases trocáveis do solo até a camada de $0,20 \mathrm{~m}$, nos diferentes sistemas de manejo em comparação ao CN (Tabela 3). Alleoni et al. (2005) relataram que a calagem, na superfície ou com incorporação, não influencia a correção da acidez do subsolo, na camada de 0,20-0,40 m, em sistema sob PD. Matias et al. (2009) verificaram, no cerrado do Piauí, que a elevação nos valores de SB decorre principalmente da prática de correção e adubação na camada arável até $0,20 \mathrm{~m}$.

Entre os sistemas de manejo, houve redução nos teores de $\mathrm{Ca}^{2+}, \mathrm{Mg}^{2+}$ e em $\mathrm{SB}$, no período posterior à colheita (seco), provavelmente em razão da exportação dos nutrientes pela cultura da soja (Tabela 3). Quanto aos teores de $\mathrm{Ca}^{2+}$, foram observados no PD9 efeitos significativos até a camada de $0,10-0,20 \mathrm{~m}$, com exceção do período seco, no qual PC3 destacou-se, possivelmente em consequência da incorporação de calcário nessa camada. A aplicação de $\mathrm{Ca}^{2+}{\mathrm{e} \mathrm{Mg}^{2+}}^{2+}$

Tabela 2. Valores de $\mathrm{pH}$ em água $(1: 2,5)$, alumínio $\left(\mathrm{Al}^{3+}\right)$, hidrogênio+alumínio $(\mathrm{H}+\mathrm{Al})$ e percentagem de saturação por alumínio $(\mathrm{m} \%)$ de Latossolo Amarelo sob cinco sistemas de manejo, em dois períodos de coleta (chuvoso e seco) e quatro camadas de profundidade ${ }^{(1)}$.

\begin{tabular}{|c|c|c|c|c|c|c|c|c|}
\hline \multirow[t]{2}{*}{ Sistema } & \multicolumn{2}{|c|}{$0,00-0,05 \mathrm{~m}$} & \multicolumn{2}{|c|}{$0,05-0,10 \mathrm{~m}$} & \multicolumn{2}{|c|}{$0,10-0,20 \mathrm{~m}$} & \multicolumn{2}{|c|}{$0,20-0,40 \mathrm{~m}$} \\
\hline & Chuvoso & Seco & Chuvoso & Seco & Chuvoso & Seco & Chuvoso & Seco \\
\hline & \multicolumn{8}{|c|}{$\mathrm{pH}$ em água } \\
\hline $\mathrm{CN}$ & $4,79 \mathrm{c}$ & $4,87 \mathrm{c}$ & $4,85 \mathrm{~d}$ & $4,88 \mathrm{~b}$ & $5,06 \mathrm{a}$ & $5,01 \mathrm{a}$ & $5,17 \mathrm{a}$ & $5,08 \mathrm{a}$ \\
\hline PC3 & $5,32 b$ & $5,20 \mathrm{ab}$ & $5,22 \mathrm{ab}$ & $4,97 \mathrm{ab}$ & $4,90 \mathrm{a}$ & $4,92 \mathrm{a}$ & $4,39 \mathrm{~cd}$ & $4,50 \mathrm{c}$ \\
\hline PD3 & $5,38 \mathrm{ab}$ & $5,28 \mathrm{a}$ & $5,01 \mathrm{~cd}$ & $5,01 \mathrm{ab}$ & $4,70 \mathrm{~b}$ & $5,00 \mathrm{a}$ & $4,26 \mathrm{~d}$ & $4,83 b$ \\
\hline PD5 & $5,25 b$ & $5,07 \mathrm{~b}$ & $5,06 \mathrm{bc}$ & $4,87 \mathrm{~b}$ & $4,92 \mathrm{a}$ & $4,86 \mathrm{a}$ & $4,50 \mathrm{bc}$ & $4,82 \mathrm{~b}$ \\
\hline \multirow[t]{2}{*}{ PD9 } & $5,53 \mathrm{a}$ & $5,14 \mathrm{ab}$ & $5,26 \mathrm{a}$ & $5,08 \mathrm{a}$ & $4,91 \mathrm{a}$ & $5,01 \mathrm{a}$ & $4,66 \mathrm{~b}$ & $4,86 \mathrm{~b}$ \\
\hline & \multicolumn{8}{|c|}{$\mathrm{Al}^{3+}\left(\mathrm{cmol}_{\mathrm{c}} \mathrm{dm}^{-3}\right)$} \\
\hline $\mathrm{CN}$ & $2,07 \mathrm{a}$ & $2,52 \mathrm{a}$ & $1,69 \mathrm{a}$ & $2,06 \mathrm{a}$ & $1,32 \mathrm{a}$ & $1,52 \mathrm{a}$ & $0,95 \mathrm{a}$ & $1,12 b$ \\
\hline PC3 & $0,52 b$ & $0,59 b$ & $0,47 \mathrm{c}$ & $1,36 b$ & $0,57 b$ & $1,29 \mathrm{a}$ & $0,89 \mathrm{a}$ & $1,49 \mathrm{a}$ \\
\hline PD3 & $0,24 b c$ & $0,45 b$ & $0,87 \mathrm{~b}$ & $1,20 \mathrm{~b}$ & $0,78 \mathrm{~b}$ & $1,33 \mathrm{a}$ & $0,72 \mathrm{a}$ & $1,10 \mathrm{~b}$ \\
\hline PD5 & $0,20 \mathrm{c}$ & $0,43 b$ & $0,69 b c$ & $1,35 b$ & $0,82 \mathrm{~b}$ & $1,36 \mathrm{a}$ & $0,74 \mathrm{a}$ & $1,27 \mathrm{ab}$ \\
\hline \multirow[t]{2}{*}{ PD9 } & $0,17 \mathrm{c}$ & $0,57 \mathrm{~b}$ & $0,51 \mathrm{c}$ & $1,21 \mathrm{~b}$ & $0,82 \mathrm{~b}$ & $1,29 \mathrm{a}$ & $0,71 \mathrm{a}$ & $1,11 \mathrm{~b}$ \\
\hline & \multicolumn{8}{|c|}{$\mathrm{H}+\mathrm{Al}\left(\mathrm{cmol}_{\mathrm{c}} \mathrm{dm}^{-3}\right)$} \\
\hline $\mathrm{CN}$ & 9,41 a & $9,65 \mathrm{a}$ & $6,27 b$ & $8,45 \mathrm{a}$ & $5,28 \mathrm{c}$ & $6,30 \mathrm{ab}$ & $4,06 \mathrm{ab}$ & $4,08 \mathrm{~b}$ \\
\hline PC3 & $5,31 \mathrm{~d}$ & $7,65 b$ & $5,84 \mathrm{bc}$ & $8,02 \mathrm{ab}$ & $5,15 \mathrm{c}$ & $7,17 \mathrm{a}$ & $3,20 \mathrm{~b}$ & $5,30 \mathrm{a}$ \\
\hline PD3 & $4,32 d$ & $7,63 b$ & $4,91 \mathrm{c}$ & $8,08 \mathrm{ab}$ & $6,00 \mathrm{bc}$ & $5,11 \mathrm{c}$ & $4,29 \mathrm{ab}$ & $3,83 b$ \\
\hline PD5 & $8,12 b$ & $7,28 b$ & $8,38 \mathrm{a}$ & $8,37 \mathrm{ab}$ & $7,19 \mathrm{a}$ & $6,33 \mathrm{ab}$ & $5,15 \mathrm{a}$ & $4,59 \mathrm{ab}$ \\
\hline \multirow[t]{2}{*}{ PD9 } & $6,53 \mathrm{c}$ & $6,80 \mathrm{~b}$ & $6,90 \mathrm{~b}$ & $7,21 b$ & $6,86 \mathrm{ab}$ & $5,81 \mathrm{bc}$ & $4,35 \mathrm{ab}$ & $4,31 \mathrm{ab}$ \\
\hline & \multicolumn{8}{|c|}{ Saturação por alumínio (\%) } \\
\hline $\mathrm{CN}$ & $83,39 a$ & $88,59 a$ & $91,78 \mathrm{a}$ & $95,74 a$ & $93,95 \mathrm{a}$ & $95,99 a$ & $91,99 a$ & $95,70 \mathrm{a}$ \\
\hline PC3 & $17,74 b$ & $24,01 b$ & $16,78 \mathrm{c}$ & $43,71 b$ & $23,85 \mathrm{~d}$ & $50,59 d$ & $72,60 b$ & $87,01 \mathrm{~b}$ \\
\hline PD3 & $7,00 \mathrm{c}$ & $16,35 \mathrm{c}$ & $33,38 b$ & $49,40 \mathrm{~b}$ & $52,18 b$ & $74,10 \mathrm{~b}$ & $70,83 \mathrm{bc}$ & $84,31 b c$ \\
\hline PD5 & $4,54 \mathrm{c}$ & $13,78 \mathrm{c}$ & $20,23 \mathrm{c}$ & $42,23 b$ & $37,52 \mathrm{c}$ & $66,44 c$ & $69,21 \mathrm{bc}$ & $81,75 b c$ \\
\hline PD9 & $3,71 \mathrm{c}$ & $18,56 \mathrm{bc}$ & $13,94 \mathrm{c}$ & $42,55 b$ & $36,82 \mathrm{c}$ & $65,85 \mathrm{c}$ & $64,53 c$ & $79,24 \mathrm{c}$ \\
\hline
\end{tabular}

${ }^{(1)}$ Médias seguidas de letras iguais, nas colunas, não diferem entre si, pelo teste Tukey, a 5\% de probabilidade. CN, cerrado nativo; PC3, plantio convencional por três anos; PD3, plantio direto por três anos; PD5, plantio direto por cinco anos; PD9, plantio direto por nove anos. 
de forma superficial em sistemas de PD diminui a eficiência na correção da acidez em profundidade, em razão das características de baixa solubilidade desses elementos (Flores et al., 2008). Os teores de $\mathrm{Mg}^{2+}$ foram superiores, o que indica melhor distribuição desse cátion até a camada de $0,20 \mathrm{~m}$ no sistema PC3. Isto se deve à incorporação do calcário dolomítico realizada em sistema convencional de cultivo, que promove uma

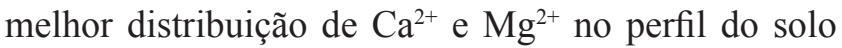
(Matias et al., 2009).

Houve baixo consumo de $\mathrm{K}^{+}$(Tabela 3), possivelmente em razão do deficit hídrico ocorrido após a sua aplicação em cobertura, realizada 27 dias após a semeadura. O PD9 apresentou maiores valores de teores de $\mathrm{K}^{+}$entre os sistemas. Garcia et al. (2008) ressaltaram que espécies forrageiras, quando usadas como plantas de cobertura, são eficientes na extração e ciclagem de $\mathrm{K}^{+}$no solo, em sistemas de rotação de culturas. A baixa abundância de compostos orgânicos em PC3 pode ter proporcionado a lixiviação de $\mathrm{K}^{+}$no períodos chuvoso, o que resultou na redução nos teores desse cátion de 0,32 na seca para $0,17 \mathrm{cmol}_{\mathrm{c}} \mathrm{dm}^{-3}$ nas águas, na camada de $0,00-0,05 \mathrm{~m}$, e de 0,15 para $0,09 \mathrm{cmol}_{\mathrm{c}} \mathrm{dm}^{-3}$, na camada de 0,05-0,10 m. Maior perda de nutrientes pode estar associada a sistemas de manejo que eliminam os resíduos do solo (Goedert \& Oliveira, 2007).

Observou-se melhor distribuição de $\mathrm{Ca}^{2+}, \mathrm{Mg}^{2+}$ e K em PD9, na camada de até $0,10 \mathrm{~m}$, e PC3, na camada de $0,10-0,20$ m (Tabela 3 ).

Valores superiores de CTC efetiva foram observados nos sistemas PD5 e PD9, até a camada de 0,10-0,20 m, exceto no período seco (Tabela 4). De acordo com Ciotta et al. (2003), apesar de baixo, o acúmulo de matéria orgânica na superfície do solo, com argila de atividade baixa em sistema PD, resulta em importante aumento nos valores de CTC efetiva e T, com melhores resultados até $8 \mathrm{~cm}$ de profundidade. Comportamento similar foi observado no PC 3 , na camada de $0,05-0,10$ e $0,20-0,40$, no período seco, e na de $0,10-0,20 \mathrm{~m}$, em ambos os períodos.

Tabela 3. Teores de cálcio, magnésio, potássio e soma de bases (SB) de Latossolo Amarelo sob cinco sistemas de manejo, em dois períodos de coleta (chuvoso e seco) e quatro profundidades ${ }^{(1)}$.

\begin{tabular}{|c|c|c|c|c|c|c|c|c|}
\hline \multirow[t]{2}{*}{ Sistema } & \multicolumn{2}{|c|}{$0,00-0,05 \mathrm{~m}$} & \multicolumn{2}{|c|}{$0,05-0,10 \mathrm{~m}$} & \multicolumn{2}{|c|}{$0,10-0,20 \mathrm{~m}$} & \multicolumn{2}{|c|}{$0,20-0,40 \mathrm{~m}$} \\
\hline & Chuvoso & Seco & Chuvoso & Seco & Chuvoso & Seco & Chuvoso & Seco \\
\hline & \multicolumn{8}{|c|}{$\mathrm{Ca}^{2+}\left(\mathrm{cmol}_{\mathrm{c}} \mathrm{dm}^{-3}\right)$} \\
\hline $\mathrm{CN}$ & $0,19 \mathrm{e}$ & $0,14 \mathrm{c}$ & $0,03 \mathrm{~d}$ & $0,02 \mathrm{c}$ & $0,02 \mathrm{c}$ & $0,02 \mathrm{c}$ & $0,03 \mathrm{a}$ & $0,02 \mathrm{a}$ \\
\hline PC3 & $1,53 \mathrm{~d}$ & $1,11 \mathrm{~b}$ & $1,52 \mathrm{c}$ & $1,15 \mathrm{ab}$ & $1,22 \mathrm{a}$ & $0,85 \mathrm{a}$ & $0,19 \mathrm{a}$ & $0,09 \mathrm{a}$ \\
\hline PD3 & $2,19 \mathrm{c}$ & $1,40 \mathrm{~b}$ & $1,37 \mathrm{c}$ & $0,87 b$ & $0,58 b$ & $0,31 b c$ & $0,22 \mathrm{a}$ & $0,12 \mathrm{a}$ \\
\hline PD5 & $3,25 b$ & $1,86 \mathrm{a}$ & $2,32 b$ & $1,45 \mathrm{a}$ & $1,18 \mathrm{a}$ & $0,49 \mathrm{~b}$ & $0,26 \mathrm{a}$ & $0,18 \mathrm{a}$ \\
\hline \multirow[t]{2}{*}{ PD9 } & $3,66 \mathrm{a}$ & $1,90 \mathrm{a}$ & $2,70 \mathrm{a}$ & $1,24 \mathrm{a}$ & $1,19 \mathrm{a}$ & $0,45 b$ & $0,30 \mathrm{a}$ & $0,20 \mathrm{a}$ \\
\hline & \multicolumn{8}{|c|}{$\mathrm{Mg}^{2+}\left(\mathrm{cmol}_{\mathrm{c}} \mathrm{dm}^{-3}\right)$} \\
\hline $\mathrm{CN}$ & $0,15 \mathrm{~d}$ & $0,13 \mathrm{c}$ & $0,05 \mathrm{~d}$ & $0,03 \mathrm{c}$ & $0,03 \mathrm{c}$ & $0,02 b$ & $0,03 \mathrm{a}$ & $0,02 \mathrm{a}$ \\
\hline $\mathrm{PC} 3$ & $0,69 \mathrm{a}$ & $0,50 \mathrm{a}$ & $0,72 \mathrm{a}$ & $0,44 \mathrm{a}$ & $0,55 \mathrm{a}$ & $0,31 \mathrm{a}$ & $0,04 \mathrm{a}$ & $0,10 \mathrm{a}$ \\
\hline PD3 & $0,65 \mathrm{ab}$ & $0,59 \mathrm{a}$ & $0,17 \mathrm{c}$ & $0,16 b$ & $0,07 b c$ & $0,07 b$ & $0,03 \mathrm{a}$ & $0,03 \mathrm{a}$ \\
\hline PD5 & $0,56 b c$ & $0,44 b$ & $0,20 \mathrm{bc}$ & $0,17 \mathrm{~b}$ & $0,09 \mathrm{bc}$ & $0,07 \mathrm{~b}$ & $0,03 \mathrm{a}$ & $0,04 \mathrm{a}$ \\
\hline \multirow[t]{2}{*}{ PD9 } & $0,52 \mathrm{c}$ & $0,36 \mathrm{~b}$ & $0,28 \mathrm{~b}$ & $0,21 b$ & $0,13 b$ & $0,09 \mathrm{~b}$ & $0,04 \mathrm{a}$ & $0,04 \mathrm{a}$ \\
\hline & \multicolumn{8}{|c|}{$\mathrm{K}^{+}\left(\mathrm{cmol}_{\mathrm{c}} \mathrm{dm}^{-3}\right)$} \\
\hline $\mathrm{CN}$ & $0,05 \mathrm{~d}$ & $0,04 \mathrm{c}$ & $0,06 \mathrm{~b}$ & $0,03 \mathrm{c}$ & $0,02 b$ & $0,02 \mathrm{c}$ & $0,03 \mathrm{a}$ & $0,02 \mathrm{a}$ \\
\hline $\mathrm{PC} 3$ & $0,17 \mathrm{c}$ & $0,32 b$ & $0,09 \mathrm{~b}$ & $0,15 b$ & $0,06 a b$ & $0,05 \mathrm{bc}$ & $0,02 \mathrm{a}$ & $0,02 \mathrm{a}$ \\
\hline PD3 & $0,35 b$ & $0,36 b$ & $0,20 \mathrm{a}$ & $0,18 \mathrm{ab}$ & $0,06 \mathrm{ab}$ & $0,07 \mathrm{ab}$ & $0,03 \mathrm{a}$ & $0,05 \mathrm{a}$ \\
\hline PD5 & $0,38 b$ & $0,41 \mathrm{a}$ & $0,20 \mathrm{a}$ & $0,21 \mathrm{a}$ & $0,09 \mathrm{a}$ & $0,10 \mathrm{a}$ & $0,03 \mathrm{a}$ & $0,04 \mathrm{a}$ \\
\hline \multirow[t]{2}{*}{ PD9 } & $0,43 \mathrm{a}$ & $0,40 \mathrm{a}$ & $0,22 \mathrm{a}$ & $0,20 \mathrm{a}$ & $0,09 \mathrm{a}$ & $0,11 \mathrm{a}$ & $0,04 \mathrm{a}$ & $0,04 \mathrm{a}$ \\
\hline & \multicolumn{8}{|c|}{$\mathrm{SB}\left(\mathrm{cmol}_{\mathrm{c}} \mathrm{dm}^{-3}\right)$} \\
\hline $\mathrm{CN}$ & $0,40 \mathrm{e}$ & $0,32 \mathrm{c}$ & $0,15 \mathrm{e}$ & $0,09 \mathrm{c}$ & $0,08 \mathrm{~d}$ & $0,06 \mathrm{c}$ & $0,09 \mathrm{a}$ & $0,06 \mathrm{a}$ \\
\hline PC3 & $2,41 d$ & $1,93 b$ & $2,34 \mathrm{c}$ & $1,74 a$ & $1,84 \mathrm{a}$ & $1,21 \mathrm{a}$ & $0,25 \mathrm{a}$ & $0,22 \mathrm{a}$ \\
\hline PD3 & $3,20 \mathrm{c}$ & $2,35 \mathrm{a}$ & $1,75 \mathrm{~d}$ & $1,22 b$ & $0,72 \mathrm{c}$ & $0,46 b$ & $0,29 a$ & $0,20 \mathrm{a}$ \\
\hline PD5 & $4,19 b$ & $2,72 \mathrm{a}$ & $2,73 b$ & $1,84 \mathrm{a}$ & $1,37 \mathrm{~b}$ & $0,67 b$ & $0,33 \mathrm{a}$ & $0,26 \mathrm{a}$ \\
\hline PD9 & $4,63 \mathrm{a}$ & $2,66 \mathrm{a}$ & $3,21 \mathrm{a}$ & $1,65 \mathrm{a}$ & $1,42 \mathrm{~b}$ & $0,66 \mathrm{~b}$ & $0,39 \mathrm{a}$ & $0,29 \mathrm{a}$ \\
\hline
\end{tabular}

${ }^{(1)}$ Médias seguidas de letras iguais, nas colunas, não diferem entre si, pelo teste Tukey, a 5\% de probabilidade. CN, cerrado nativo; PC3, plantio convencional por três anos; PD3, plantio direto por três anos; PD5, plantio direto por cinco anos; PD9, plantio direto por nove anos. 
Os sistemas com mais de cinco anos de adoção do plantio direto apresentaram os maiores valores de capacidade de troca cationnica potencial, em todas as camadas, exceto no período seco, nas camadas de $0,00-0,05,0,05-0,10$ e $0,10-0,20 \mathrm{~m}$, em que PC3 entre os sistemas com maiores valores de $\mathrm{T}$ (Tabela 4). Isto pode ser atribuído à estabilização da MOS entre os sistemas, nestas camadas, já que as percentagens de saturação por base $(\mathrm{V})$ do sistema PD5 variaram entre as profundidades e os períodos. Essa contribuição de CTC nem sempre está relacionada positivamente à saturação por bases, uma vez que os íons que geram a acidez do solo são cátions que podem ocupar as cargas negativas existentes na superfície do solo. Assim, as bases que são trocadas pelos íons $\mathrm{H}^{+}$e $\mathrm{Al}^{3+}$ permanecem na solução do solo e ficam suscetíveis à lixiviação (Matias et al., 2009).

A saturação por bases variou de $1,07 \%$, no $\mathrm{CN}$, a 42,56 \%, no PD3, com maiores diferenças na primeira camada (Tabela 4). A calagem favorece o aumento de $\mathrm{V}$ e a diminuição de $\mathrm{m} \%$; porém, a redução dos teores de $\mathrm{Al}^{3+}$ trocável nem sempre está associada à saturação por bases pela calagem, mas sim pela complexação do $\mathrm{Al}^{3+}$ por compostos orgânicos estáveis. Os resultados colocam em evidência a possibilidade de se reduzir o uso de adubos e corretivos para o cultivo da soja no cerrado do Piauí. No ano agrícola 2009/2010, houve aplicação de uma menor quantidade de calcário aos sistemas com maior tempo sob PD.

Os teores de COT foram superiores, no período chuvoso, nos diferentes sistemas de manejo do solo, em comparação com $\mathrm{CN}$, possivelmente em consequência da maior presença de raízes e de resíduos vegetais em processo de decomposição (Tabela 5). Todavia, as altas temperaturas no período seco aceleraram a mineralização da matéria orgânica do solo e podem ter contribuído para aumentar a perda de $\mathrm{C}$ dessas frações mais lábeis no solo.

$\mathrm{Na}$ camada de 0,00-0,05 $\mathrm{m}$, o solo sob $\mathrm{CN}$ apresentou maior teor de COT (Tabela 5), tanto no período chuvoso $\left(30,65 \mathrm{~g} \mathrm{~kg}^{-1}\right)$ como no período seco $\left(21,82 \mathrm{~g} \mathrm{~kg}^{-1}\right)$. Os sistemas PD3 e PC3 apresentaram os menores valores de COT (16,58 e $17,95 \mathrm{~g} \mathrm{~kg}^{-1}$, respectivamente) no período chuvoso, na camada superficial, em que o PC3 apresentou o menor valor $\left(12,04 \mathrm{~g} \mathrm{~kg}^{-1}\right)$ no período seco. Os baixos teores de COT observados nesses sistemas de manejo, na camada de 0,00-0,05 m, estão relacionados ao curto período de adoção do plantio direto em PD3 e ao preparo do solo em PC3.

Tabela 4. Valores da capacidade de troca catiônica efetiva e potencial, e percentagem de saturação por bases de Latossolo Amarelo sob cinco sistemas de manejo, em dois períodos de coleta (chuvoso e seco) e quatro profundidades ${ }^{(1)}$.

\begin{tabular}{|c|c|c|c|c|c|c|c|c|}
\hline \multirow[t]{2}{*}{ Sistema } & \multicolumn{2}{|c|}{$0,00-0,05 \mathrm{~m}$} & \multicolumn{2}{|c|}{$0,05-0,10 \mathrm{~m}$} & \multicolumn{2}{|c|}{$0,10-0,20 \mathrm{~m}$} & \multicolumn{2}{|c|}{$0,20-0,40 \mathrm{~m}$} \\
\hline & Chuvoso & Seco & Chuvoso & Seco & Chuvoso & Seco & Chuvoso & Seco \\
\hline & \multicolumn{8}{|c|}{ Capacidade de troca catiônica efetiva $\left(\mathrm{cmol}_{\mathrm{c}} \mathrm{dm}^{-3}\right)$} \\
\hline $\mathrm{CN}$ & $2,47 d$ & $2,84 \mathrm{ab}$ & $1,84 \mathrm{c}$ & $2,15 b$ & $1,40 \mathrm{~b}$ & $1,58 \mathrm{c}$ & $1,03 \mathrm{a}$ & $1,17 b$ \\
\hline PC3 & $2,93 \mathrm{c}$ & $2,47 b$ & $2,81 b$ & $3,11 \mathrm{a}$ & $2,41 \mathrm{a}$ & $2,50 \mathrm{a}$ & $1,22 \mathrm{a}$ & $1,71 \mathrm{a}$ \\
\hline PD3 & $3,44 b$ & $2,80 \mathrm{ab}$ & $2,62 b$ & $2,42 b$ & $1,50 \mathrm{~b}$ & $1,80 \mathrm{bc}$ & $1,01 \mathrm{a}$ & $1,30 \mathrm{ab}$ \\
\hline PD5 & $4,39 \mathrm{a}$ & $3,15 \mathrm{a}$ & $3,42 \mathrm{a}$ & $3,19 \mathrm{a}$ & $2,19 a$ & $2,04 b$ & $1,07 \mathrm{a}$ & $1,55 \mathrm{ab}$ \\
\hline \multirow[t]{2}{*}{ PD9 } & $4,80 \mathrm{a}$ & $3,19 \mathrm{a}$ & $3,72 \mathrm{a}$ & $2,87 \mathrm{a}$ & $2,24 \mathrm{a}$ & $1,96 \mathrm{bc}$ & $1,10 \mathrm{a}$ & $1,40 \mathrm{ab}$ \\
\hline & \multicolumn{8}{|c|}{ Capacidade de troca catiônica potencial $\left(\mathrm{cmol}_{\mathrm{c}} \mathrm{dm}^{-3}\right)$} \\
\hline $\mathrm{CN}$ & $9,80 \mathrm{~b}$ & $9,97 \mathrm{a}$ & $6,41 \mathrm{c}$ & $8,54 \mathrm{c}$ & $5,36 \mathrm{c}$ & $6,10 \mathrm{bc}$ & $4,14 b c$ & $4,13 b$ \\
\hline PC3 & $7,72 \mathrm{c}$ & $9,53 \mathrm{a}$ & $8,18 b$ & $9,77 \mathrm{ab}$ & $6,99 b$ & $8,39 \mathrm{a}$ & $3,54 \mathrm{c}$ & $5,52 \mathrm{a}$ \\
\hline PD3 & $7,52 \mathrm{c}$ & $9,99 \mathrm{a}$ & $6,67 \mathrm{c}$ & $9,50 \mathrm{bc}$ & $6,72 b$ & $5,57 \mathrm{c}$ & $4,60 \mathrm{bc}$ & $4,03 \mathrm{~b}$ \\
\hline PD5 & $12,31 \mathrm{a}$ & $10,00 \mathrm{a}$ & $11,11 \mathrm{a}$ & $10,21 \mathrm{a}$ & $8,56 a$ & $7,00 \mathrm{~b}$ & $5,47 \mathrm{a}$ & $4,87 \mathrm{ab}$ \\
\hline \multirow[t]{2}{*}{ PD9 } & $11,16 \mathrm{a}$ & $9,42 \mathrm{a}$ & $10,11 \mathrm{a}$ & $8,87 \mathrm{bc}$ & $8,28 \mathrm{a}$ & $6,47 \mathrm{bc}$ & $4,74 \mathrm{ab}$ & $4,60 \mathrm{ab}$ \\
\hline & \multicolumn{8}{|c|}{ Saturação por bases $(\%)$} \\
\hline $\mathrm{CN}$ & $4,23 \mathrm{c}$ & $3,24 \mathrm{c}$ & $2,35 \mathrm{c}$ & $1,07 \mathrm{c}$ & $1,57 d$ & $1,04 \mathrm{c}$ & $2,04 \mathrm{~b}$ & $1,23 b$ \\
\hline PC3 & $31,32 b$ & $19,78 b$ & $28,65 \mathrm{ab}$ & $17,96 \mathrm{a}$ & $26,65 \mathrm{a}$ & $14,52 \mathrm{a}$ & $9,55 \mathrm{a}$ & $4,03 \mathrm{ab}$ \\
\hline PD3 & $42,56 a$ & $23,71 \mathrm{ab}$ & $26,30 \mathrm{~b}$ & $13,18 b$ & $10,66 \mathrm{c}$ & $8,38 b$ & $6,42 \mathrm{a}$ & $5,01 \mathrm{ab}$ \\
\hline PD5 & $34,15 b$ & $27,57 \mathrm{a}$ & $24,76 b$ & $18,25 \mathrm{a}$ & $16,15 b$ & $9,67 \mathrm{~b}$ & $6,02 \mathrm{ab}$ & $5,69 \mathrm{a}$ \\
\hline PD9 & $41,32 \mathrm{a}$ & $27,75 a$ & $31,67 \mathrm{a}$ & $18,66 \mathrm{a}$ & $17,18 \mathrm{~b}$ & $10,29 \mathrm{ab}$ & $8,18 \mathrm{a}$ & $6,27 \mathrm{a}$ \\
\hline
\end{tabular}

${ }^{(1)}$ Médias seguidas de letras iguais, nas colunas, não diferem entre si, pelo teste Tukey, a 5\% de probabilidade. CN, cerrado nativo; PC3, plantio convencional por três anos; PD3, plantio direto por três anos; PD5, plantio direto por cinco anos; PD9, plantio direto por nove anos. 
Tabela 5. Teores de carbono orgânico total e fósforo disponível de Latossolo Amarelo sob cinco sistemas de manejo, em dois períodos de coleta (chuvoso e seco) e quatro profundidades ${ }^{(1)}$.

\begin{tabular}{|c|c|c|c|c|c|c|c|c|}
\hline \multirow[t]{2}{*}{ Sistema } & \multicolumn{2}{|c|}{$0,00-0,05 \mathrm{~m}$} & \multicolumn{2}{|c|}{$0,05-0,10 \mathrm{~m}$} & \multicolumn{2}{|c|}{$0,10-0,20 \mathrm{~m}$} & \multicolumn{2}{|c|}{$0,20-0,40 \mathrm{~m}$} \\
\hline & Chuvoso & Seco & Chuvoso & Seco & Chuvoso & Seco & Chuvoso & Seco \\
\hline & \multicolumn{8}{|c|}{ Carbono orgânico total $\left(\mathrm{g} \mathrm{kg}^{-1}\right)$} \\
\hline $\mathrm{CN}$ & $30,65 a$ & $21,82 \mathrm{a}$ & $18,09 \mathrm{~b}$ & $9,99 \mathrm{c}$ & $13,39 \mathrm{a}$ & $7,59 \mathrm{c}$ & $9,30 \mathrm{a}$ & $5,77 \mathrm{bc}$ \\
\hline PC3 & $17,95 \mathrm{c}$ & $12,04 d$ & $18,43 b$ & $12,07 b$ & $15,14 \mathrm{a}$ & $8,35 \mathrm{c}$ & $9,18 \mathrm{a}$ & $4,91 \mathrm{c}$ \\
\hline PD3 & $16,58 \mathrm{c}$ & $17,58 \mathrm{c}$ & $18,45 b$ & $13,38 b$ & $11,01 b$ & $7,56 \mathrm{c}$ & $7,98 \mathrm{a}$ & $7,06 \mathrm{ab}$ \\
\hline PD5 & $22,03 b$ & $20,10 \mathrm{ab}$ & $21,81 \mathrm{a}$ & $18,79 \mathrm{a}$ & $15,03 \mathrm{a}$ & $10,81 b$ & $9,89 \mathrm{a}$ & $8,73 \mathrm{a}$ \\
\hline \multirow[t]{2}{*}{ PD9 } & $20,41 b$ & $18,94 \mathrm{bc}$ & $18,68 \mathrm{~b}$ & $17,61 \mathrm{a}$ & $15,20 \mathrm{a}$ & $12,89 \mathrm{a}$ & $8,80 \mathrm{a}$ & $8,86 a$ \\
\hline & \multicolumn{8}{|c|}{ Fósforo $\left(\mathrm{mg} \mathrm{dm}^{-3}\right)$} \\
\hline $\mathrm{CN}$ & $1,54 \mathrm{~d}$ & $2,07 \mathrm{~d}$ & $1,11 \mathrm{e}$ & $1,38 \mathrm{~d}$ & $0,86 \mathrm{c}$ & $1,00 \mathrm{c}$ & $0,86 \mathrm{a}$ & $1,02 \mathrm{a}$ \\
\hline $\mathrm{PC} 3$ & $13,76 \mathrm{c}$ & $11,37 \mathrm{c}$ & $5,66 \mathrm{~d}$ & $8,96 \mathrm{c}$ & $4,39 b c$ & $4,30 \mathrm{bc}$ & $0,77 \mathrm{a}$ & $1,13 \mathrm{a}$ \\
\hline PD3 & $44,55 b$ & $36,24 b$ & $23,41 \mathrm{~b}$ & $31,31 \mathrm{a}$ & $3,75 b c$ & $8,00 \mathrm{~b}$ & $0,50 \mathrm{a}$ & $1,66 a$ \\
\hline PD5 & $42,74 b$ & $39,08 \mathrm{~b}$ & $17,15 \mathrm{c}$ & $14,95 b$ & $6,41 \mathrm{~b}$ & $7,24 b$ & $1,00 \mathrm{a}$ & $1,51 \mathrm{a}$ \\
\hline PD9 & $66,12 \mathrm{a}$ & $48,88 \mathrm{a}$ & $41,37 \mathrm{a}$ & $29,54 a$ & $17,68 \mathrm{a}$ & $15,17 \mathrm{a}$ & $1,31 \mathrm{a}$ & $2,37 \mathrm{a}$ \\
\hline
\end{tabular}

${ }^{(1)}$ Médias seguidas de letras iguais, nas colunas, não diferem entre si, pelo teste Tukey, a 5\% de probabilidade. CN, cerrado nativo; PC3, plantio convencional por três anos; PD3, plantio direto por três anos; PD5, plantio direto por cinco anos; PD9, plantio direto por nove anos.

Nas camadas de $0,05-0,10,0,10-0,20$ e $0,20-0,40 \mathrm{~m}$, exceto no período chuvoso para a última camada, os sistemas PD5 e PD9 foram superiores na comparação dos teores de COT. No entanto, a semelhança dos valores de COT, na camada de $0,10-0,20$ m no período chuvoso entre PC3, PD5 e PD9 pode estar relacionada ao uso de implementos agrícolas utilizados antes da implantação do plantio direto. Balesdent et al. (2000) relataram que o arado de aiveca, utilizado em plantio convencional, incorpora resíduos em profundidade e transloca a MOS para profundidades abaixo do pé-de-arado. Observou-se diferença significativa nos teores de COT entre os sistemas PD3, PD5 e PD9, na camada de $0,20-0,40 \mathrm{~m}$, no período seco, o que mostra que a presença de resíduos vegetais, aliada ao não revolvimento do solo e à rotação de culturas, pode causar acúmulo de $\mathrm{C}$ no perfil do solo.

O sistema PD9 apresentou valores superiores de P disponível, nos períodos chuvoso e seco, até a camada de $0,20 \mathrm{~m}$ (Tabela 5). O plantio direto formado pela cobertura de diferentes espécies vegetais tem a capacidade de acumular certos nutrientes, como $\mathrm{P}$ e $\mathrm{K}^{+}$. Este efeito é mais evidente na superfície do solo (Santos et al., 2009). Todavia, a melhor distribuição do P disponível ao longo do perfil no PD9 pode estar relacionada à presença do sistema radicular da braquiária, que favorece a formação de um horizonte orgânico de resíduos vegetais de lenta decomposição. Para elementos com baixa taxa de difusão no solo, como os fosfatos, plantas com maior superfície radicular, como as gramíneas, têm maior capacidade de absorção desse nutriente (Garcia et al., 2008). A disponibilidade de $\mathrm{P}$ variou entre os períodos avaliados (Tabela 5), porém, no período seco, foi maior nos sistemas PC3 e PD3, nas camadas de 0,05-0,10 m e 0,10-0,20 m, provavelmente em razão da remoção das bases do solo pela cultura (Viégas et al., 2010).

\section{Conclusões}

1. Após cinco anos, o plantio direto proporciona aumento nos teores $\mathrm{Ca}^{2+}, \mathrm{K}^{+}, \mathrm{P}$ e na soma de bases, até a profundidade de $0,20 \mathrm{~m}$.

2. O aumento nos teores de carbono orgânico total, em sistemas sob plantio direto no cerrado do Piauí, contribui para a redução dos componentes da acidez do solo e o aumento da capacidade de troca catiônica até a profundidade de $0,20 \mathrm{~m}$.

3. O plantio direto, associado ao uso de forrageiras, favorece o acúmulo de carbono orgânico nas camadas mais profundas do solo, principalmente no período seco.

\section{Agradecimentos}

À Coordenação de Aperfeiçoamento de Pessoal de Nível Superior, por concessão de bolsa; ao Conselho Nacional de Desenvolvimento Científico e Tecnológico e à Fundação de Amparo à Pesquisa do Estado do Piauí, pelo apoio financeiro; e à família Maggioni, pela cessão das áreas experimentais. 


\section{Referências}

AGUIAR, T. de J.A. de; MONTEIRO, M. do S.L. Modelo agrícola e desenvolvimento sustentável: a ocupação do Cerrado piauiense. Ambiente e Sociedade, v.8, p.1-18, 2005.

ALLEONI, L.R.F.; CAMBRI, M.A.; CAIRES, E.F. Atributos químicos de um Latossolo de Cerrado sob plantio direto, de acordo com doses e formas de aplicação de calcário. Revista Brasileira de Ciência do Solo, v.29, p.923-934, 2005.

ANGHINONI, I. Fertilidade do solo e seu manejo em sistema plantio direto. In: NOVAIS, R.F.; ALVAREZ V., V.H.; BARROS, N.F. de; FONTES, R.L.F.; CANTARUTTI, R.B.; NEVES, J.C.L. (Ed.). Fertilidade do solo. Viçosa: Sociedade Brasileira de Ciência do Solo, 2007. p.873-928.

BALESDENT, J.; CHENU, C.; BALABANE, M. Relationship of soil organic matter dynamics to physical protection and tillage. Soil and Tillage Research, v.53, p.215-230, 2000.

BALOTA, E.L.; KANASHIRO, M.; COLOZZI FILHO, A.; ANDRADE, D.S.; DICK, R.P. Soil enzyme activities under long-term tillage and crop rotation systems in subtropical agro-ecosystems. Brazilian Journal of Microbiology, v.35, p.300-306, 2004.

BOER, C.A.; ASSIS, R.L. de; SILVA, G.P.; BRAZ, A.J.B.P.; BARROSO, A.L. de L.; CARGNELUTTI FILHO, A.; PIRES, F.R. Ciclagem de nutrientes por plantas de cobertura na entressafra em um solo de Cerrado. Pesquisa Agropecuária Brasileira, v.42, p.1269-1276, 2007.

CARNEIRO, M.A.C.; SOUZA, E.D. de; REIS, E.F. dos; PEREIRA, H.S.; AZEVEDO, W.R. de. Atributos físicos, químicos e biológicos de solo de Cerrado sob diferentes sistemas de uso e manejo. Revista Brasileira de Ciência do Solo, v.33, p.147-157, 2009.

CIOTTA, M.N.; BAYER, C.; FONTOURA, S.M.V.; ERNANI, P.R.; ALBUQUERQUE, J.A. Matéria orgânica e aumento da capacidade de troca de cátions em solo com argila de atividade baixa sob plantio direto. Ciência Rural, v.33, p.1161-1164, 2003.

FLORES, J.P.C.; CASSOL, L.C.; ANGHINONI, I.; CARVALHO, P.C. de F. Atributos químicos do solo em função da aplicação superficial de calcário em sistema integração lavoura-pecuária submetidos a pressões de pastejo em plantio direto. Revista Brasileira de Ciência do Solo, v.32, p.2385-2396, 2008.

FONTANA, A.; PEREIRA, M.G.; LOSS, A.; CUNHA, T.J.F.; SALTON, J.C. Atributos de fertilidade e frações húmicas de um Latossolo Vermelho no Cerrado. Pesquisa Agropecuária Brasileira, v.41, p.847-853, 2006.

GARCIA, R.A.; CRUSCIOL, C.A.C.; CALONEGO, J.C.; ROSOLEM, C.A. Potassium cycling in a corn-brachiaria cropping system. European Journal of Agronomy, v.28, p.579-585, 2008.

GATIBONI, L.C.; KAMINSKI, J.; RHEINHEIMER, D. dos S.; FLORES, J.P.C. Biodisponibilidade de formas de fósforo acumuladas em solo sob sistema plantio direto. Revista Brasileira de Ciência do Solo, v.31, p.691-699, 2007.

GOEDERT, W.J.; OLIVEIRA, S.A. Fertilidade do solo e sustentabilidade da atividade agrícola. In: NOVAIS, R.F.;
ALVAREZ V., V.H.; BARROS, N.F. de; FONTES, R.L.F.; CANTARUTTI, R.B.; NEVES, J.C.L. (Ed.). Fertilidade do solo. Viçosa: Sociedade Brasileira de Ciência do Solo, 2007. p.991-1017.

LOPES, A.S.; WIETHÖLTER, S.; GUILHERME, L.R.G.; SILVA, C.A. Sistema plantio direto: bases para o manejo da fertilidade do solo. São Paulo: ANDA, 2004. 115p. Disponível em: $<$ http:// www.anda.org.br/livrostecnicos/lt_spd.pdf $>$. Acesso em: 11 set. 2011.

MARCHÃO, R.L.; LAVELLE, P.; CELINE, L.; BALBINO, L.C.; VILELA, L.; BECQUER, T. Soil macrofauna under integrated crop-livestock systems in a Brazilian Cerrado Ferralsol. Pesquisa Agropecuária Brasileira, v.44, p.1011-1020, 2009.

MATIAS, M. da C.B.; SALVIANO, A.A.C.; LEITE, L.F.C.; GALVÃO, S.R. da S. Propriedades químicas em Latossolo Amarelo de Cerrado do Piauí sob diferentes sistemas de manejo. Revista Ciência Agronômica, v.40, p.356-362, 2009.

NICOLOSO, R. da S.; LOVATO, T.; CARNEIRO AMADO, T.J.; BAYER, C.; LANZANOVA, M.E. Balanço do carbono orgânico no solo sob integração lavoura-pecuária no Sul do Brasil. Revista Brasileira de Ciência do Solo, v.32, p.2425-2433, 2008.

NOLLA, A.; ANGHINONI, I. Critérios de calagem para a soja no sistema plantio direto consolidado. Revista Brasileira de Ciência do Solo, v.30, p.475-483, 2006.

PERIN, E.; CERETTA, C.A.; KLAMT, E. Tempo de uso agrícola e propriedades químicas de dois Latossolos do Planalto Médio do Rio Grande do Sul. Revista Brasileira de Ciência do Solo, v.27, p.665-674, 2003.

RIBEIRO, A.C.; GUIMARAES, P.T.G.; ALVAREZ V., V.H. (Ed.). Recomendações para o uso de corretivos e fertilizantes em Minas Gerais: $5^{\text {a }}$ aproximação. Viçosa: Comissão de Fertilidade do Solo do Estado de Minas Gerais, 1999. 359p.

ROSOLEM, C.A.; CALONEGO, J.C.; FOLONI, J.S.S. Lixiviação de potássio da palha de espécies de cobertura de solo de acordo com a quantidade de chuva aplicada. Revista Brasileira de Ciência do Solo, v.27, p.355-362, 2003.

SALTON, J.C.; MIELNICZUK, J.; BAYER, C.; BOENI, M.; CONCEIÇÃO, P.C.; FABRICIO, A.C.; MACEDO, M.C.M.; BROCH, D.L. Agregação e estabilidade de agregados do solo em sistemas agropecuários em Mato Grosso do Sul. Revista Brasileira de Ciência do Solo, v.32, p.11-21, 2008.

SANTOS, H.G. dos; JACOMINE, P.K.T.; ANJOS, L.H.C. dos; OLIVEIRA, V.A. de; OLIVEIRA, J.B.; COELHO, M.R.; LUMBRERAS, J.F.; CUNHA, T.J.F. (Ed.). Sistema brasileiro de classificação de solos. 2.ed. Rio de Janeiro: Embrapa Solos, 2006. $306 \mathrm{p}$.

SANTOS, H.P. dos; FONTANELI, R.S.; SPERA, S.T.; TOMM, G.O. Efeito de sistemas de produção integração lavoura-pecuária (ILP) sobre a fertilidade do solo em plantio direto. Acta Scientiarum. Agronomy, v.31, p.719-727, 2009.

SILVA, F.C. da (Org.). Manual de análises químicas de solos, plantas e fertilizantes. Brasília: Embrapa Comunicação para Transferência de Tecnologia, 1999. 370p. 
SILVA, F. de A.S. e.; AZEVEDO, C.A.V. de. Principal components analysis in the software Assistat-Statistical Attendance. In: WORLD CONGRESS ON COMPUTERS IN AGRICULTURE, 7., 2009, Reno. Proceedings. Michigan: American Society of Agricultural and Biological Engineers, 2009.

SOUSA, D.M.G.; MIRANDA, L.N.; OLIVEIRA, S.A. Acidez do solo e sua correção. In: NOVAIS, R.F.; ALVAREZ V., V.H.; BARROS, N.F. de; FONTES, R.L.F.; CANTARUTTI, R.B.; NEVES, J.C.L. (Ed.). Fertilidade do solo. Viçosa: Sociedade Brasileira de Ciência do Solo, 2007. p.205-274.
VIÉGAS, R.A.; NOVAIS, R.F.; SCHULTHAIS, F. Availability of a soluble phosphorus source applied to soil samples with different acidicity levels. Revista Brasileira de Ciência do Solo, v.34, p.1126-1136, 2010.

YEOMANS, J.C.; BREMNER, J.M. A rapid and precise method for routine determination of organic carbon in soil. Communications in Soil Science and Plant Analysis, v.19, p.1467-1476, 1988.

ZAMBROSI, F.C.B; ALLEONI, L.R.F.; CAIRES, E.F. Liming and ionic speciation of an Oxisol under no-till system. Scientia Agricola, v.65, p.190-203, 2008.

Recebido em 3 de março de 2011 e aprovado em 18 de novembro de 2011 